\title{
LÉOPARD EST UN CHAT AUSSI
}

\author{
Anna BoCHNAKOWA \\ Université Jagellonne
}

\begin{abstract}
The article contains an overview of Polish and French verbs derived from animal names. The etymology and meaning of the following verbs are given:

- in Polish: lampartować się, bobrować, byczyć się, chomikować, dzięciolić, jeżyć się, koziołkować, kozłować, matpować, mrowić, mrowić się, myszkować, naindyczyć się, osowieć, przekomarzać się, psieć, rozwydrzyć, sępić, zasępić się, stchórzyć, ślimaczyć się, świnić, świnić się, wyjelenić, zacietrzewić się, zbaranieć;

- in French: ânonner, s'avachir, bicher, bichonner, cafarder, canarder, cavaler, chatoyer, chevaucher, chevroter, cochonner, s'encanailler, faisander, fouiner, fourmiller, fureter, grenouiller, se hérisser, lézarder, louveter, louvoyer, méduser, miter, moutonner, paonner, se pavaner, papillonner, pigeonner, punaiser, rater, renarder, renauder, seriner, serpenter, singer, tigrer, zébrer.
\end{abstract}

\section{INTRODUCTION}

C'est le mot polonais lampartować się, évoqué par Alicja, qui a été le point de départ de la présente contribution. Puisque je ne le connaissais pas, la Jubilaire m'a expliqué qu'il figurait dans le texte de Moralność pani Dulskiej ${ }^{1}$ de Gabriela Zapolska (publié en 1907) et désignait la conduite peu louable du jeune Dulski qui découche souvent et séduit la servante. Je n'ai pas retenu le mot de cette lecture obligatoire au lycée, et c'est maintenant que je le découvre.

\footnotetext{
${ }^{1}$ Fr. 'La morale de Madame Dulska'. « Czy ty się przestaniesz lampartować? » ('Vas-tu arrêter de *te léoparder ?') - demande Mme Dulska à son fils rentrant le matin après la nuit de débauche (Acte I, scène V, p. 11).
} 


\section{Du LÉOPARD}

Le nom lampart 'léopard' est noté en polonais depuis le XVI ${ }^{\mathrm{e}}$ siècle (Stownik polszczyzny XVI wieku). Le sens figuré 'człowiek lampart, włóczęga, basałyk, do niczego' ('homme léopard, vagabond, voyou, vaurien') a été mentionné dans le dictionnaire du polonais de Linde (1807). Le dictionnaire dit Wileński (Zdanowicz et al., 1861) donne le verbe lampartować 'prowadzić życie hultajskie, rozwiązłe' et lampartować się (réfléchi) 'usposabiać się na lamparta, włóczęgę, szlifobruka' ('devenir lampart, vagabond, batteur de pavé'). Stownik języka polskiego (Karłowicz, Kryński, Niedźwiedzki, 1900) note lampartować et lamparcić się 'być łobuzem, hultajem, włóczęga, brukotłukiem, hulaka, birbantem' ('être voyou, vagabond, batteur de pavé...'). Vers le milieu du XX $\mathrm{XX}^{\mathrm{e}}$ le verbe lampartować (się) 'prowadzić życie rozpustne, hulaszcze, próżniacze' ('mener une vie dissolue, oisive') est considéré comme vieilli dans le volumineux dictionnaire de polonais de Doroszewski (1958). Slownik języka polskiego PWN de 2016 (en ligne) mentionne encore le verbe lampartować się 'mener une vie dissolue, débauchée' et le qualifie aussi de vieilli. Dans le contemporain Wielki stownik języka polskiego le mot n'est pas répertorié, et le NKJP (Corpus National de la Langue Polonaise ${ }^{2}$ ) donne une seule attestation de 2006.

Ayant appris l'origine du sens du beau mot lampartować, lié au sens figuré de lampart 'léopard' ('vagabond, voyou, vaurien'), noté au début du XIX', je ne savais toujours pas quel est le rapport de cet animal exotique avec une vie de voyou et de vagabond. Serait-ce un mode de vie de l'animal, n'apparaissant pas en Pologne, donc sortant de l'ordinaire, vivant et chassant souvent en solitaire ? Mais je n'ai aucun appui pour cette supposition, sauf le principe de l'étymologie dite populaire... Un éclaircissement pourtant se trouve dans un article de Kleczkowski (1921). L'auteur se penche sur l'origine mystérieuse de ce verbe et du nom lampart 'voyou' et il propose (Kleczkowski, 1921 : 108) de rapporter le mot polonais à lump, du même sens, provenant de l'allemand Lump (Lumpen) 'chiffon', d'origine néerlandaise, de lomperd, devenu en allemand Lumpert 'vagabond, voyou'. Cette forme empruntée par le polonais serait rapprochée par une étymologie populaire de lampart 'léopard', probablement uniquement à cause de ressemblance formelle. Kleczkowski écarte le lien avec Lombard dans le sens 'usurier', proposé avant par quelques chercheurs (mentionnés par Kleczkowski, ibid., p. 107). Bien que l'histoire de lampart 'voyou, vagabond' ne soit pas tracée d'une façon absolument probante, j'admets volontiers le rapprochement fait en polonais par l'étymologie populaire, qui faute de mieux, justifie le sens de lampartować.

\footnotetext{
${ }^{2}$ http://nkjp.pl.
} 


\section{VERBES PROVENANT DES NOMS D'ANIMAUX}

Inspirée par cet exemple intéressant de dérivation et d'évolution sémantique, j'ai décidé de passer en revue les verbes polonais et français provenant des noms d'animaux.

Dans les deux langues nous avons des verbes formés à partir de noms d'animaux, tels que : pol. kocić się, cielić się ('mettre bas : à propos de chattes et de vaches') ou fr. pouiller 'chercher des poux', vêler, lapiner ou encore toréer, mais ils se distinguent par un lien sémantique qui résulte de l'implication réelle de l'animal dans l'action nommée. C'est pour cela que je les exclus de ma liste de verbes où le nom d'animal est évoqué à titre symbolique, stéréotypé ou fantaisiste.

Je n'ai pas l'intention de donner ici une étude comparative des unités particulières dans les deux langues, je voudrais juste les répertorier et observer leur signification. Je passerai donc en ordre alphabétique d'abord les verbes polonais, puis français. J'ai constitué mon modeste corpus m'appuyant uniquement sur ma compétence linguistique dans les deux langues ${ }^{3}$. Dans mon inventaire, j'expliquerai uniquement l'étymologie des mots polonais à côté de leur sens ${ }^{4}$, en estimant que les formes françaises sont généralement connues et leur étymologie claire. Je tâcherai aussi, tant bien que mal, car intuitivement, de donner la justification métaphorique des verbes.

\subsection{VERBES POLONAIS}

Bobrować 'fouiner, fouiller à la recherche de quelque chose', de bóbr 'castor'. La motivation sémantique du verbe est due probablement aux mouvements agiles de l'animal qui s'occupe en permanence à amasser des branches dans l'eau pour en faire des barrages et des abris...

Byczyć się fam. 'paresser, se reposer', de byk 'taureau'. L'aspect du mâle imposant, couché et inerte fait-il penser à un repos paisible, sans envie aucune de bouger et d'agir?

${ }^{3}$ Et profitant de l'amabilité de la famille et des amis qui me soufflaient leurs exemples. Et je les remercie tous ! Que mes remerciements particuliers aillent vers Jean-Pierre Darcel, lecteur de français dans mon Institut qui a entrepris la recherche sur internet.

${ }^{4}$ En principe, je donne les verbes dans leur forme imperfective, à moins que celle-ci ne soit pas utilisée, laissant place à la perfective. Il arrive aussi que les deux fonctionnent, mais avec des sens différents. Je me réfère aux définitions succinctes de Slownik języka polskiego PWN 2016 (en ligne https://sjp.pwn.pl, voir Pałka et Kwaśnicka, 2017 : 90) ou celles de Slownik języka polskiego, ortograficzny, wyrazów obcych i slownik do gier w jednym (en ligne https://sjp.pl/). 
Chomikować fam. 'mettre de côté, garder quelque chose sans besoin apparent, faire des provisions souvent inutiles', de chomik 'hamster'. Le verbe met en valeur l'action de transporter de la nourriture dans les abajoues pour la porter dans un endroit sûr et en faire des provisions.

Dzięciolić 'apprendre par cœur, bachoter', de dzięciol 'pivert'. Bachoter en polonais se dit zakuwać, kuć 'taper comme le fait un pivert', ce qui dans l'argot scolaire veut dire 'apprendre beaucoup, souvent par cœur et sans réflexion'. Et la répétition par cœur rappellerait le tapage monotone du pivert.

Jeżyć się 1. 'se dresser' en parlant des cheveux ; 2. 's'élever, en parlant des obstacles ou des difficultés' ; 3. 'être méfiant, suspicieux, ne pas désirer la communication', de jeż 'hérisson'. Les deux premières acceptions se réfèrent à l'aspect des poils durcis d'un hérisson, le troisième sens illustre métaphoriquement l'attitude de refus de contact, la protection contre l'entourage, la méfiance.

Koziolkować 'cabrioler', de koziolek 'cabri', diminutif de koziol 'bouc' (de koza 'chèvre'). L'action semble rappeler les sauts de cabri, de chèvre.

Kozłować 'faire rebondir le ballon, dans le jeu de basket ou de hand-ball', de koziot 'bouc'. Ce sont les rebonds du ballon qui sont comparés aux sauts de bouc / chèvre.

Matpować 'singer', de matpa 'singe'. Puisqu'on voit dans les gestes et les grimaces de singes l'imitation (ou simplement la similitude) du comportement humain, le nom de cet animal a servi, dans les deux langues d'ailleurs, à la formation du verbe qui désigne l'action d'imiter quelqu'un, parfois en le ridiculisant.

Mrowić 'engourdir, avoir la chair de poule', de mrówka 'fourmi'. La sensation d'engourdissement, de contraction de la peau est comparée à l'impression imaginaire de passage massif de fourmis dans notre corps.

Mrowić się 1. 'en parlant d'une foule réunie dans un endroit et se déplaçant dans des sens divers'; 2. 'en parlant des endroits où les hommes ou les animaux bougent en masse', de mrówka 'fourmi', insecte qui vit en colonies complexes et toujours en mouvement.

Myszkować 'fouiner, chercher furtivement', de mysz 'souris'. Cette action est comparée à la recherche de la nourriture ou simplement à l'inspection des lieux de ce rongeur s'invitant dans les demeures humaines. 
Naindyczyć się fam. 'montrer la mauvaise humeur ou la colère', de indyk 'dindon'. On peut supposer que le visage rougi de colère peut ressembler à la tête de dinde ornée de caroncules de cette couleur et le glougloutement de l'oiseau fait penser aux cris de mécontentement.

Osowiec' 'devenir triste, apathique', de sowa 'hibou', souvent confondu avec la chouette. La tête de ce rapace nocturne aux yeux perçants, immobilisé aux aguets peut donner l'image d'un être sérieux, perdu dans ses pensées. D'ailleurs, la chouette symbolisant la sagesse renvoie à une certaine tenue.

Przekomarzać się 'taquiner quelqu'un en plaisantant', de komar 'moustique'. Le fait d'agacer quelqu'un gentiment et sans méchanceté est comparé à une piqûre d'un moustique.

Psieć en plaisantant 'perdre de l'importance, de la valeur, se détériorer, se gâcher', de pies 'chien'. Ce verbe reste certainement en rapport avec le sens des adjectifs psi, pieski 'médiocre, triste, piètre, quelconque' ('de chien').

Rozwydrzyć 'laisser quelqu'un devenir désobéissant, capricieux', rozwydrzyć się 'devenir désobéissant, gâté', de wydra 'loutre'. La seule explication que je pourrais donner pour justifier le lien entre le sens du verbe et le nom de l'animal serait la mention d'une loutre domestiquée (appelée Robak) qui appartenait à un noble polonais Jan Chryzostom Pasek (vers 1636-1701) et puis a été offerte, au grand regret du gentilhomme, au roi Jean III Sobieski. Pasek, un homme extravagant et aventurier ${ }^{5}$, est considéré comme un représentant caractéristique de la Pologne nobiliaire du XVII ${ }^{e}$ siècle. On sait que la loutre de Pasek était très gâtée par son maître, qu'elle l'accompagnait dans ses déplacements et constituait une curiosité de société.

Sepić fam. 1. 'demander d'une façon insistante, extorquer de l'argent'; 2. 'lésiner, ne pas accorder', de sęp 'vautour'. Le verbe semble faire référence à la force et à la cruauté du charognard, aussi bien dans le sens de demander sans refus possible que d'être inflexible et sourd aux besoins et aux attentes d'autrui.

Zasępić się 'prendre un air morose, s'assombrir', de sęp 'vautour'. Est-ce la renommée peu favorable de l'oiseau qui se nourrit de charogne qui fait imaginer sa morosité ?

\footnotetext{
${ }^{5}$ Ses Pamiętniki ('Mémoires') sont une source souvent exploitée par les historiens polonais.
} 
Stchórzyć 'reculer par peur devant un obstacle, un danger, agir lâchement', de tchórz 'putois'. Puisque le putois réagit à la peur ou à la menace par l'éjection d'un liquide puant, sa lâcheté présumée devient visible et il est facile de motiver le sens du verbe formé à partir du nom de putois par son comportement naturel.

Ślimaczyć się 1. 'suinter, en parlant des plaies' ; 2. 'avancer très lentement, aussi au sens figuré', de ślimak 'escargot'. Ces sens différents s'appuient sur deux images que l'on peut associer à l'escargot. Premièrement, c'est l'aspect gluant de sa bave à laquelle on compare une plaie qui ne cicatrise pas. Dans le deuxième cas, à l'origine de la métaphore se trouve la lenteur avec laquelle se déplace un escargot sur son pied. Ce verbe se rapporte par exemple à des procédures ou à des affaires qui traînent excessivement.

Świnić 1. fam. 'salir, mettre en désordre' ; 2. fam. 'agir ou parler d'une façon obscène'.

Świnić się 1. fam. 'se salir'; 2. fam. 'devenir un homme sans principes, immoral', de świnia 'cochon'. On attribue à cet animal le goût de la saleté ou au moins on considère la porcherie comme un endroit malodorant, malpropre, sali de nourriture et d'excréments. Il est donc facile d'utiliser son nom pour créer un verbe synonyme de salir, et pour nommer le comportement grossier, on utilise aussi le même verbe. La forme réfléchie, dans le deuxième sens, se rapporte à une attitude "sale " de quelqu'un qui ne respecte pas les valeurs et les principes moraux vis-à-vis des autres.

Wyjelenić fam. 'duper, tromper', de jeleń 'cerf'. Le verbe reste en rapport avec le sens de jeleń dans les expressions familières znaleźć jelenia ('trouver un naïf'), robić z kogoś jelenia ('duper, tromper quelqu'un'), où ce jeleń est pris au figuré pour 'trompé, cocu'.

Zacietrzewić się 's'emporter dans une discussion et rester intransigeant', de cietrzew 'tétras lyre'. Le sens du verbe vient probablement de la comparaison d'un homme emporté par la colère à l'aspect d'un tétras en parade nuptiale, déroulant la queue, roucoulant fort, imposant et perdu dans sa passion.

Zbaranieć fam. 'devenir pantois, interloqué, stupéfait', de baran 'mouton'. Puisqu'on attribue la bêtise aux moutons (au moins en Pologne), le fait d'être interloqué, surpris par un propos ou une situation nous abêtit, nous abrutit et nous rend semblable à un mouton... 
Il faut constater que les verbes cités ci-dessus témoignent d'une créativité lexicale bien imagée et se référant à des animaux bien connus qui nous sont familiers.

\subsection{VERBES FRANÇAIS}

Le français offre aussi une vision pittoresque de nos actes, en renvoyant aux autres animaux. En voici des verbes formés à partir des noms d'animaux ${ }^{6}$ :

Ânonner pour 'lire ou réciter un texte d'une façon laborieuse et monotone'.

(S) 'avachir pour 'perdre sa forme, devenir mou' et 's'affaler'. Effectivement, le gros corps d'une vache peut paraître informe, et un homme affalé dans un fauteuil peut l'être aussi...

Bicher pop. 'être content, se réjouir'. Il est vrai que les biches, gracieuses dans leurs mouvements donnent l'impression de joie et de jeu !

Bichonner fam. 'parer quelqu'un, un enfant, avec une recherche coquette ou le choyer'. Le verbe illustre les soins et la tendresse que l'on voit dans l'attitude d'un maître ou d'une maîtresse envers son bichon, petit chien d'agrément à fourrure abondante et museau court (TLFi).

Cafarder vient du sens familier de cafard 'mouchard'. Le nom de cet insecte dégoûtant donné à un dénonciateur exprime le mépris de la personne?

Canarder signifie 'tirer en étant caché'. Quand on tire sur des canards qui nagent sur des étangs, il vaut mieux être caché, par exemple dans des tonnes flottantes au bord des mares où les canards se posent. C'est une condition indispensable dans cette chasse à terrain découvert, où l'on serait vite repéré par les proies.

Cavaler veut dire étymologiquement 'aller à cheval', donc plus rapidement qu'à pied, d'où probablement le sens de 'courir'.

Chatoyer, pour parler des étoffes ou des pierres précieuses, provient de chat. Le $T L F$ informatisé explique que le dérivé se justifie par la comparaison de l'aspect de ces objets aux reflets changeants de l'œil de l'animal.

\footnotetext{
${ }^{6}$ Les définitions de sens viennent - si ce n'est pas marqué autrement - du Dictionnaire Larousse en ligne : http://www.larousse.fr/dictionnaires.
} 
Chevaucher - l'étymologie dit clairement que le verbe signifie 'aller à cheval', sans pourtant insister sur la vitesse du déplacement comme c'est le cas dans cavaler, dérivé, lui aussi, du latin vulgaire caballus 'cheval'.

Chevroter renvoie simplement par son sens de 'parler d'une voix tremblante' à la voix de chèvre...

Cochonner fam. 1. 'faire salement, grossièrement quelque chose' ; 2. 'salir, abîmer'. Le cochon n'a pas de bonne réputation...

(S) 'encanailler provient en fin de compte de canis 'chien' en latin. Le fait de s'avilir, de prendre les manières vulgaires renvoie finalement à une vie de chien qui ne paraît pas fameuse, par ailleurs.

Faisander, pour parler de la viande, est lié au fait du traitement particulier du gibier à poil et à plumes, par l'exposition prolongée au froid pour faire attendrir la viande avant la consommation.

Fouiner pour 'fouiller dans les affaires ou dans la vie de quelqu'un pour y découvrir quelque chose de secret' attribue l'indiscrétion à la fouine qui se glisse dans les poulaillers par les plus petites ouvertures.

Fourmiller - la motivation du sens paraît évidente, il suffit de regarder une fourmilière.

Fureter renvoie à une pratique de chasse au lapin où l'on utilisait des furets pour débusquer la proie. D'où le sens de 'chercher soigneusement pour découvrir quelque chose de caché, de secret'. On y retrouve la proximité sémantique avec fouiner.

Grenouiller intrigue par son sens de 'intriguer plus ou moins malhonnêtement auprès des uns et des autres' et le lien formel avec la grenouille. Est-ce le coassement de grenouilles qui symbolise les cancans animés par les malveillants?

(Se) hérisser, du même sens que le mot polonais jeżyć się, s'appuie sur la même métaphore construite sur l'aspect de l'animal pourvu de poils raides, semblant inaccessible, comme quelqu'un de hérissé. 
Lézarder 'former des fissures sur un mur', est un dérivé direct de lézarde 'fissure', lui-même provenant de lézard. Un mur abîmé semblerait couvert de lézards qui aiment bien se poser sur une surface ensoleillée, d'où aussi le sens généralement connu de paresser au soleil...

Louveter est lié au sens figuré du mot loup 'une machine servant à démêler la laine avant le tissage' qui est comparée à l'animal aux dents puissantes.

Louvoyer vient de l'image d'un loup qui suit son chemin en faisant des détours et le verbe exprime l'action de biaiser, tergiverser pour atteindre son but.

Méduser provient plutôt du nom propre mythique, mais puisqu'il est aussi celui d'un animal, on peut l'inclure dans cet inventaire de fortune. Et puisqu'il veut dire 'frapper quelqu'un de stupeur' et que l'on sait que le contact avec les tentacules de certaines méduses peut paralyser leur proie, le sens du verbe se voit justifié.

Miter 'pleurnicher' en argot (TLFi) serait lié au sens 'cachot' qu'avait aussi le mot mite (fém. ou masc.) d'origine incertaine. S'agit-il peut-être d'un simple homonyme du nom d'insecte?

Moutonner, en parlant de la mer, décrit l'eau agitée, formant de petites vagues blanches, ressemblant à la toison bouclée de l'animal.

Paonner reste en rapport sémantique avec faire la roue comme le fait le paon et nomme le concept de se glorifier d'une façon très réussie.

Se pavaner, venant aussi du lat. pavo 'paon', appelle d'une façon très juste le fait de se tenir d'une façon orgueilleuse, vaniteuse, d'avoir une allure visant à plaire. Effectivement, un paon faisant la roue éveille l'admiration...

Papillonner illustre bien le comportement de quelqu'un qui, comme un papillon, va d'un objet à l'autre, sans trop s'arrêter, ou s'intéresse d'une façon superficielle à plusieurs choses.

Pigeonner pour dire 'rouler, tromper quelqu'un' fait allusion au sens familier et figuré de pigeon - 'un homme naïf, facile à duper'. Visiblement, le pigeon ne passe pas pour un oiseau trop intelligent... 
Punaiser paraît clair si l'on pense au sens figuré de punaise 'une sorte de petit clou'. L'insecte n'y est pas évoqué directement.

Rater a une provenance intéressante. Le mot viendrait (TLFi) de rat dans le sens 'coup de feu qui n'est pas parti' dans prendre un rat ('ne pas partir d'une arme à feu'), datant du XVII' siècle (TLFi), d'où rater son coup 'ne pas faire feu en parlant d'une arme'. Le sens de 'échouer, ne pas réussir, manquer' s'est développé après.

Renarder 'recourir aux ruses' exploite le stéréotype du renard comme étant un animal intelligent et rusé. Le $T L F i$ donne aussi une acception populaire et vieillie - 'vomir', justifiée par le comportement de l'animal.

Renauder pop. et vieilli 'nasiller', arg. 'manifester son mécontentement, bougonner, renâcler' (TLFi). Le sens argotique viendrait de parler regnaut (variante de renard) 'nasiller'.

Seriner fam. 'répéter inlassablement quelque chose à quelqu'un' fait probablement allusion au chant répétitif d'un serin.

Serpenter se rapporte à la forme sinueuse que prend un serpent quand il se déplace.

Singer devient clair quand on observe ces animaux qui nous ressemblent...

Tigrer 'orner de raies semblables à celles sur la peau d'un tigre' est sémantiquement tout à fait clair.

Zébrer c'est l'action de marquer de rayures et le sens du verbe s'impose facilement.

\section{RÉCAPITULATION}

Tous les verbes cités font preuve de notre symbiose symbolique avec le monde animal. Nous l'observons et nous retrouvons nos attitudes dans le comportement des animaux.

Dans les exemples polonais et français sont fixés des noms d'une petite ménagerie ; il serait risqué de justifier leur composition pour chaque exemple, il est intéressant toutefois de constater la présence des uns ou des autres dans les langues 
respectives et de découvrir le sens attribué aux verbes formés à partir des noms n'animaux. Les parallélismes d'origine et de sens du verbe sont rares : je n'en n'ai trouvé que quatre paires : jeżyć się - se hérisser, matpować - singer, mrowić sięfourmiller, świnić et cochonner pour 'salir'. La plupart des verbes sont formés de noms différents, le français puise deux fois dans les noms d'animaux vivant hors de notre continent - tigrer et zébrer - du même sens d'ailleurs, ce qui peut suggérer une connaissance plus large des pays dits exotiques. Il arrive que le même sens soit attribué aux verbes provenant du nom d'animal différent dans les deux langues, comme par exemple pour le pol. myszkować (de mysz 'souris') et le fr. fouiner ou fureter pour 'fouiller, chercher d'une façon indiscrète' ou encore wyjelenić et pigeonner.

Voici ces quelques lignes, parties d'un mot polonais utilisé par Alicja ! Mais quoi qu'il en soit, on sait que le chat reste le plus important...

\section{Références bibliographiques}

\section{Ouvrages et articles}

KLECZKOWSKI, Adam (1921), «Lampart 'tobuz, birbant, hulaka' », Język Polski, VI, n 4, pp. 106-112.

PAŁKA, Patrycja, KWAŚNICKA-JANOWICZ, Agata (2017), Przewodnik po elektronicznych zasobach językowych dla polonistów, Biblioteczka Towarzystwa Miłośników Języka Polskiego, $\mathrm{n}^{\circ}$ 28, Kraków, Wydawnictwo JAK, www.tmjp.pl/images/pdf/przewodnik_po_ elektronicznych_zasobach.pdf(dernière consultation : le 14.01.2018).

PASEK, Jan Chryzostom (1836 [1690-1695]), Pamiętniki, Poznań, E. Raczyński.

ZAPOLSKA, Gabriela (1907), Moralność pani Dulskiej: komedya w trzech aktach, Warszawa, Tow. Akc. S. Orgelbranda S-ów.

\section{Dictionnaires}

Dictionnaire Larousse, www.larousse.fr/dictionnaires.

Słownik języka polskiego (1807-1814), réd. S. B. Linde, Warszawa, Drukarnia XX. Pijarów, www. kpbc.umk.pl/publication/8173 (dernière consultation : le 14.01.2018).

Słownik języka polskiego (1861), réd. A. Zdanowicz et al., Wilno, M. Olgerbrand, www.eswil.ijp. pan.pl (dernière consultation : le 07.01.2018).

Słownik języka polskiego (1900-1927), réd. J. Karłowicz, A. Kryński, W. Niedźwiedzki, Warszawa, www.ebuw.uw.edu.pl/publication/254 (dernière consultation : le 14.01.2018).

Slownik języka polskiego (1958-1969), réd. W. Doroszewski, Warszawa, Wiedza Powszechna/PWN, www.doroszewski.pwn.pl.

Stownik języka polskiego (1982), réd. M. Szymczak, Warszawa, PWN. 
Stownik języka polskiego PWN (2016), www.sjp.pwn.pl.

Stownik języka polskiego, ortograficzny, wyrazów obcych i stownik do gier w jednym, www.sjp.pl. Stownik polszczyzny XVI wieku (1956), Wrocław, Zakład im. Ossolińskich, Wydawnictwo PAN, www.kpbc.umk.pl/dlibra/publication?id=17781\&tab=1 (dernière consultation : le 12.01.2018).

Trésor de la langue française informatisé, www.atilf.atilf.fr.

Wielki słownik języka polskiego (2007), réd. P. Żmigrodzki et al., IJP PAN, www.wsjp.pl.

\section{Sitographie}

NKJP (Narodowy Korpus Języka Polskiego), www.nkjp.pl. 